\title{
Response to Wang et al.
}

W. rect a typographical error in our commentary (1) "MEF2A sequence variants and coronary heart disease: a change of heart?". Specifically, the number of nonsynonymous changes found in controls should have read " 1 in 500," not " 0 in 500 " as in our published article. (This is because the 21-nt deletion was seen in controls.) Based on the then-available counts $(5 / 500$ in cases and $1 / 500$ in controls), the 2 -tailed $P$ value we provided $(P>0.2)$ is correct.

The accompanying letter from Weng et al. adds to and clarifies their recent study (2), providing more detailed information about ascertainment of variants in cases and controls. First, all exons were resequenced in cases, but only certain exons were resequenced in controls. Second, several additional missense changes were identified in both cases and controls. Taking these data into account, it appears that there have been 7 missense changes identified in cases and 5 in controls, including 3 found uniquely in cases, 1 found uniquely in controls, and 4 found in both groups. Moreover, the incomplete ascertainment in controls by Weng et al. (2) means that the current estimate of the frequency of $M E F 2 A$ missense mutations in controls is a lower bound; additional missense changes in controls (which might have been found by more complete resequencing) would shift the combined data in the direction away from association between MEF2A variation and cardiac disease.

Finally, we note that the major prevailing bias in the association literature is publication bias; that is, there is a greater likelihood that positive claims of association will be published as compared with studies that provide no evidence of association (3). Counteracting this bias requires that we encourage the publication and dispassionate evaluation of all relevant data, whether or not the data support an association.

\section{David Altshuler ${ }^{1,2,3}$ and Joel N. Hirschhorn'1,3,4}

${ }^{1}$ Department of Genetics, Harvard Medical School, Boston, Massachusetts, USA. ${ }^{2}$ Department of Molecular Biology, Center for Human Genetic Research, and Diabetes Unit, Department of Medicine, Massachusetts General Hospital, Boston, Massachusetts, USA. ${ }^{3}$ Program in Medical and Popu- lation Genetics, Broad Institute of Harvard and Massachusetts Institute of Technology, Cambridge, Massachusetts, USA. ${ }^{4}$ Divisions of Genetics and Endocrinology, Children's Hospital, Boston, Massachusetts, USA.

Conflict of interest: The authors have declared that no conflict of interest exists.

Address correspondence to: David Altshuler, Department of Molecular Biology, Massachusetts General Hospital, Boston, Massachusetts, USA. E-mail: altshuler@molbio.mgh.harvard.edu. Or to: Joel N. Hirschhorn, Children's Hospital, Boston, Massachusetts 02115, USA. E-mail: joelh@broad.mit.edu.

J. Clin. Invest. 115:1401 (2005). doi:10.1172/ JCI25477.

1. Altshuler, D., and Hirschhorn, J.N. 2005. MEF2A sequence variants and coronary artery disease: a change of heart? J. Clin. Invest. 115:831-833. doi:10.1172/JCI200524715.

2. Weng, L., et al. 2005. Lack of MEF2A mutations in coronary artery disease. J. Clin. Invest. 115:1016-1020. doi:10.1172/JCI200524186.

3. Ioannidis J.P. 2003. Genetic associations: false or true? Trends Mol. Med. 9:135-138. 